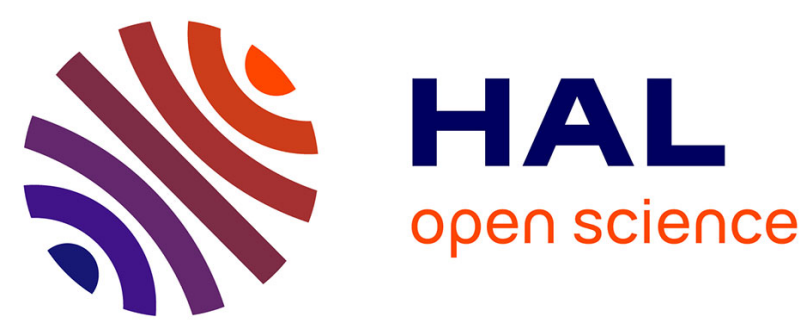

\title{
Performance of fractional delay estimation in joint estimation algorithm dedicated to digital Tx leakage compensation in FDD transceivers
}

Robin Gerzaguet, Laurent Ros, Fabrice Belvéze, Jean-Marc Brossier

\section{- To cite this version:}

Robin Gerzaguet, Laurent Ros, Fabrice Belvéze, Jean-Marc Brossier. Performance of fractional delay estimation in joint estimation algorithm dedicated to digital Tx leakage compensation in FDD transceivers. CROWNCOM 2016 - 11th EAI International Conference on Cognitive Radio Oriented Wireless Networks, EAI, May 2016, Grenoble, France. hal-01289892

\section{HAL Id: hal-01289892 \\ https://hal.science/hal-01289892}

Submitted on 17 Mar 2016

HAL is a multi-disciplinary open access archive for the deposit and dissemination of scientific research documents, whether they are published or not. The documents may come from teaching and research institutions in France or abroad, or from public or private research centers.
L'archive ouverte pluridisciplinaire HAL, est destinée au dépôt et à la diffusion de documents scientifiques de niveau recherche, publiés ou non, émanant des établissements d'enseignement et de recherche français ou étrangers, des laboratoires publics ou privés. 


\title{
Performance of fractional delay estimation in joint estimation algorithm dedicated to digital Tx leakage compensation in FDD transceivers.
}

\author{
Robin Gerzaguet $^{2}$, Laurent Ros ${ }^{1}$, Fabrice Belvéze ${ }^{3}$, and Jean-Marc Brossier ${ }^{1}$ \\ 1 GIPSA-Lab, Image and Signal Department, Saint Martin d'Hères, France, \\ ${ }^{2}$ CEA-Leti, Grenoble, France, robin.gerzaguet@cea.fr \\ 3 ST-Microelectronics, Grenoble, France
}

\begin{abstract}
This paper deals with the performance of the fractional delay estimator in the joint complex amplitude / delay estimation algorithm dedicated to digital Tx leakage compensation in FDD transceivers. Such transceivers are affected from transmitter-receiver signal leakage. Combined with non linearity of components in the received path, it leads to a pollution in the baseband signal. The baseband polluting term depends on the equivalent Tx leakage channel, modeling leakages and the received path. We have proposed in $[7,8]$ a joint estimation of the complex gain and the fractional delay and derived asymptotic performance of the complex gain estimator, that showed the necessity of the fractional delay estimation. In this paper, we propose a comprehensive study of the fractional delay estimation algorithm and its analytic performance. The study is based on the analysis of the S-curve and loop noise variance of the timing error detector, from which an approximation of the asymptotic performance of the joint estimation algorithm is derived.
\end{abstract}

Key words: Tx Leakage, FDD transceiver, Digital Compensation, Least-MeanSquare algorithm, Joint Estimation, S-curve.

\section{Introduction}

Cognitive radios offer the possibility to improve the spectrum uses and to adapt the transmission scheme to optimize the sharing of the available bandwidth, which leads to important constraints on the hardware components, located on the physical layer [1]. These constraints thus lead to performance limitations [21], and we focus on this paper on a hardware impairments that occurs when the radio follows a Frequency Division Duplexing (FDD) scheme.

Compact wireless transceivers can be based on Time Division Duplexing or on FDD modes [15] to multiplex transmission and reception links. For a frequency division duplexing framework, which is the case of the study presented here, the transmission and the reception are done simultaneously, using two different carrier frequencies.

A surface acoustic wave (SAW) duplexer is often used to connect the received 
$(\mathrm{Rx})$ and the transmitted $(\mathrm{Tx})$ path to a common antenna [11] (see figure 1). As the duplexer does not provide infinite attenuation between the $\mathrm{Rx}$ path and the Tx path, the transmitted signal can leak into the Rx path [6] leading to the so-called Tx Leakage (TxL) phenomenon. As the uplink and the downlink bands are spectrally separated, the received signal will not be directly impaired by the leakage of the transmitted signal which is filtered by the Low Pass Filter (LPF) after the demodulation.

However, due to the non linearity and imperfections of components in the analog Rx stage, especially the Low Noise Amplifier (LNA) [18] and the demodulator [14], intermodulation products can shift downward to baseband square component of the Tx Leakage signal [13]. As a consequence, this polluting signal will impair the received signal and can severely degrade the performance. The pollution is potentially detrimental in the cell edge context (i.e. when the receiver is far from the base station), where the power of the received signal is low, and the power of the transmitted signal is strong [10] leading to a strong polluting signal and thus a low signal to interference ratio.

To avoid this pollution, passive methods based on analog filtering can be imple-

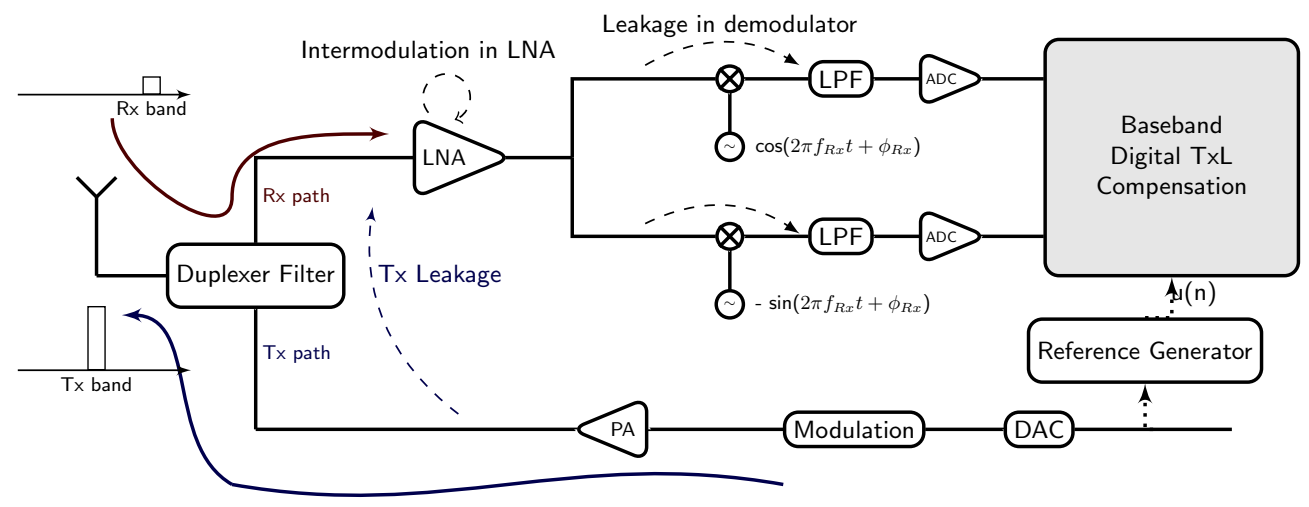

Fig. 1: Classical Frequency Division Duplexing chain in a RF Transceiver with Tx Leakage baseband pollution. The PA denotes the power Amplifier, the ADC is the Analog to Digital Converter, and the DAC denotes the Digital to Analog Converter

mented. Such mitigation methods consist in adding a band pass filter to attenuate the leaked transmitted signal in the Rx stage before or after the LNA [2]. As radio frequency ( $\mathrm{RF}$ ) transceivers contain more and more digital parts, and as signal-processing techniques are becoming an area of interest for RF impairments problematic[5], several digital compensation methods have been investigated in the past few years for the Tx Leakage (TxL) compensation $[6,10,7]$.

In this paper, we complete the performance analysis of our previously proposed joint estimation (JE) algorithm dedicated to Tx Leakage compensation [7]. This algorithm both estimates the TxL channel approximated by a time varying com- 
plex gain and the fractional delay (FD). It works with two reference based leastmean-square (LMS) algorithms. As [7] mainly focuses on the performance of the complex gain estimator (with and without a priori knowledge of the polluting FD) and not the FD nor the JE estimation algorithm itself, we focus in this paper on the FD estimation part. The two main objectives, in addition to being a comprehensive study, are to prove that the FD estimation algorithm can lock around the desired FD value and to derive an approximation of the asymptotic performance of the JE algorithm. The FD estimation algorithm has similitudes with data-aided algorithm that were designed for phase and timing synchronization as well as for automatic gain control $[16,9,19]$, however in the actual estimation process, the RX signal is considerered as noise, and an image of the Tx signal is considered as pilot datas (used as reference). In this paper, as we focus on the FD estimator, we assume a constant complex channel gain and we derive analytic formulae of the S-curve of the FD detector [16]. The S-curve represents the characteristic of the detector. For a timing detector, it describes the output of the detector with respect to the delay error between the two input components. Mathematically, it is computed as the conditional expectation of the so-called error signal that updates the FD estimation. The shape of the S-curve is an important point to understand the tracking performance of a Delay-Locked Loop [20]. Using then the general framework of the tracking loop analysis, a linear approximation of the estimation error variance of the FD stage is established from the S-curve. This result is finally used to derive an approximation of the asymptotic performance of the JE algorithm, using the primary results obtained in [7].

This paper is organized as follows. We give the baseband polluting model in section 2. We recall the joint estimation of the complex gain and the fractional delay in section 3 . We derive the performance of the fractional delay estimation algorithm in section 4 . Section 5 validates our method and theoretical results through simulations.

\section{Baseband model and issues}

The discrete time observation model sampled at $T_{\mathrm{Rx}}=1 / F_{\mathrm{Rx}}$ is expressed as

$$
d(n)=x(n)+b(n)+s_{T x L}(n)
$$

where $x(n)$ is the desired signal, which is assumed to be uncorrelated and zeromean, of variance $\sigma_{x}^{2}, b(n)$ is the white additive Gaussian noise, of variance $\sigma_{b}^{2}$, and $s_{T x L}(n)$ is the TxL polluting signal. The baseband polluting model of the TxL phenomenon is due to the cascade of several impairments, and more precisely to the combination of duplexer finite isolation, low-noise amplifier non linearity and coupling on oscillator $[6,18]$. As a consequence, the baseband polluting term can be expressed as

$$
s_{T x L}(n)=\left[h_{\mathrm{Rx}}(t) *\left|h_{\mathrm{D}}(t) * \tilde{s}_{T x}(t)\right|^{2}\right]_{t=n T_{R x}}
$$


where $h_{\mathrm{D}}$ and $h_{\mathrm{Rx}}$ denotes respectively the duplexer equivalent channel and the Rx chain equivalent channel, which models the impact of the Duplexer and the $\mathrm{Rx}$ chain, and $*$ denotes the convolution. In this paper, we suppose that the duplexer is frequency flat as this assumption is widely used in the literature $[4,6,12]$, and that the Rx filter is known (or estimated) within a complex gain introduced by the coupling at the oscillator. Besides, due to both digital and analogs blocks and propagation, the baseband polluting model is affected by a delay $\Delta$ that can be separated between an integer part $D$ of the Rx sampling time and a fractional part $\delta$. Thus, the model can be expressed as

$$
s_{T x L}(n ; \Delta)=\beta_{\mathrm{TxL}}(n)\left[\left|h_{\mathrm{D}}(t) * \tilde{s}_{T x}(t)\right|^{2}\right]_{t=(n-D) T_{R x}-\delta}
$$

where $\beta_{\mathrm{TxL}}(n)$ models the global TxL complex channel gain at time index $n$. The JE algorthm is an adaptive method based on two the LMS algorithms that can then handle time-varying parameters estimation, as presented in [7] with the tracking of the complex gain with appropriate step-size. However, as we focus on the static performance of the FD estimator in this paper, we assume a constant TxL channel for the theoretical analyis We have shown in [7] that the fractional delay cannot be neglected and we have proposed a joint estimation algorithm that is recalled in the next part. This structure is piloted by a reference signal $u(n)$, synthesized in the reference generator (see figure 1 and (4)) from the baseband Tx samples, with the known or estimated Rx filter $h_{\mathrm{Rx}}$ :

$$
u(n)=\left[h_{\mathrm{Rx}}(t) *\left|\tilde{s}_{T x}(t)\right|^{2}\right]_{t=n T_{R x}}
$$

It is to note that in practise, it may need additional processing such as an upsampler, a low pass filter, and a resampler.

\section{Joint estimation algorithm}

The structure of the joint estimation algorithm is described on figure 2. It is composed of 2 blocks, one dedicated to the complex gain estimation, with a classic one tap LMS approach and the fractional delay is estimated with another LMS where a steepest descend is applied on the FD estimation. At each iteration, the reference signal $u(n)$ is delayed of the estimated $\operatorname{FD} \delta(n)$ with an interpolated structure that can be for example a Farrow structure [3].

$$
u_{\delta_{n}}(n)=\sum_{j=0}^{L} u(n-j)\left(\prod_{\substack{i=0 \\ i \neq j}}^{L} \frac{\delta-i}{j-i}\right)
$$

The fractional delay estimation algorithm is based on the minimisation of the instantaneous square error $|e(n)|^{2}$, to which a gradient is applied. The final joint estimation algorithm can be expressed as: 


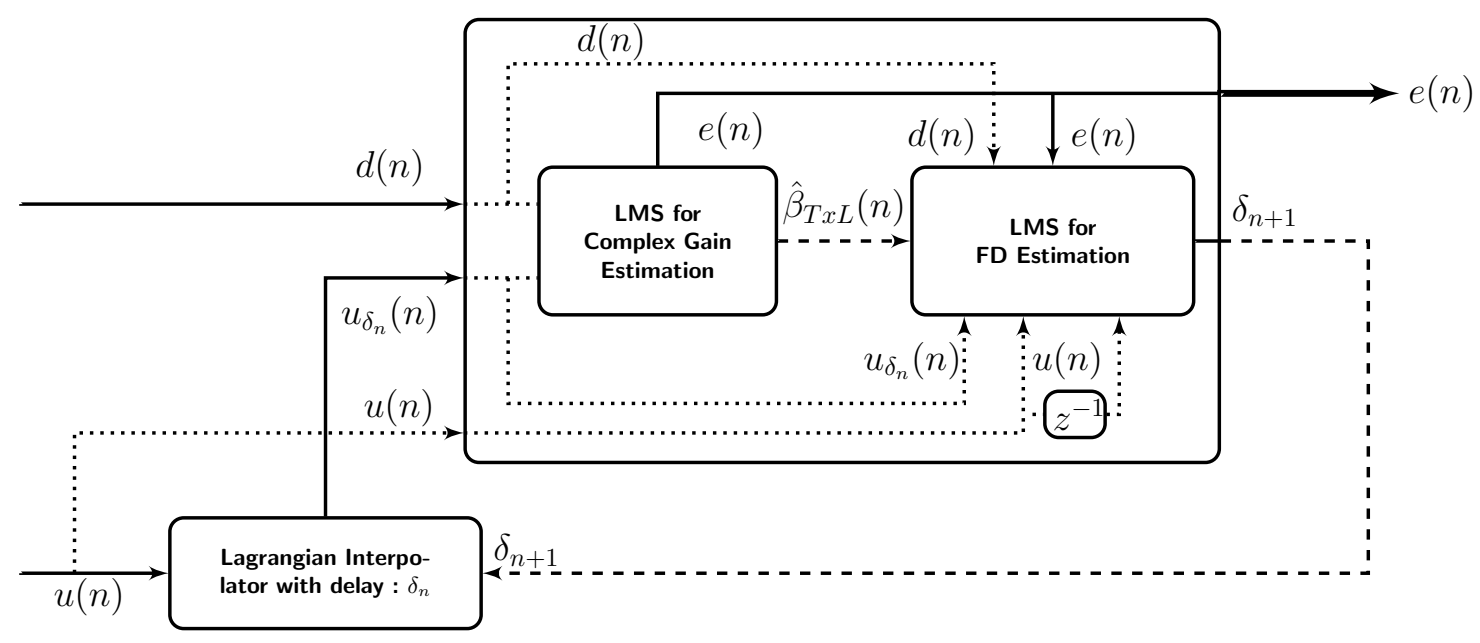

Fig. 2: Joint Estimation framework for the $T \times L$ compensation

$$
\begin{aligned}
e(n)= & d(n)-\hat{\beta}_{\mathrm{TxL}}(n) u_{\delta_{n}}(n) \\
\hat{\beta}_{\mathrm{TxL}}(n+1)= & \hat{\beta}_{\mathrm{TxL}}(n)+\mu u_{\delta_{n}}(n) e(n) \\
\Delta_{u}(n)= & u(n-1)-u(n) \\
\delta_{n+1}= & \delta_{n}+\nu \Re\left\{\left[\hat{\beta}_{T x L}(n) \Delta_{u}(n)-\right.\right. \\
& \left.\left.u_{\delta_{n}}(n) L(n)\right] e^{*}(n)\right\} \\
L(n+1)= & \left(1-\mu u_{\delta_{n}}(n)^{2}\right) L(n)+\mu d(n) \Delta_{u}(n) \\
& +2 \mu \hat{\beta}_{\mathrm{TxL}}(n) u_{\delta_{n}}(n) \Delta_{u}(n),
\end{aligned}
$$

where $e(n)$ is the compensated output, $\hat{\beta}_{\mathrm{TxL}}(n)$ the complex gain estimation, $\mu$ the step-size of the complex gain estimator, $L(n)=\partial \hat{\beta}_{\mathrm{TxL}}(n) / \partial \delta$ and $\nu$, the constant step size of the fractional delay estimator. It can be seen that this algorithm is recursive, online (as it provides a compensated output $e(n)$ at each iteration), and with low complexity.

\section{Performance of the FD estimation process}

In this section, we focus on the FD estimation algorithm. To perform the analytical study of this algorithm, we assume a perfectly known channel $\left(\hat{\beta}_{\mathrm{TxL}}(n)=\right.$ $\left.\beta_{\mathrm{TxL}}\right)$. This algorithm can be considered as a delay-locked loop synchronization algorithm and its performance can be studied with the same approach as described in $[16,20]$. Thus, the FD algorithm is equivalent to a loop algorithm (see figure 3) piloted by its error signal denoted $e_{\delta}(n)$. The stability and the performance of the proposed FD estimation algorithm can be studied using classical error detector open-loop analysis tools. 


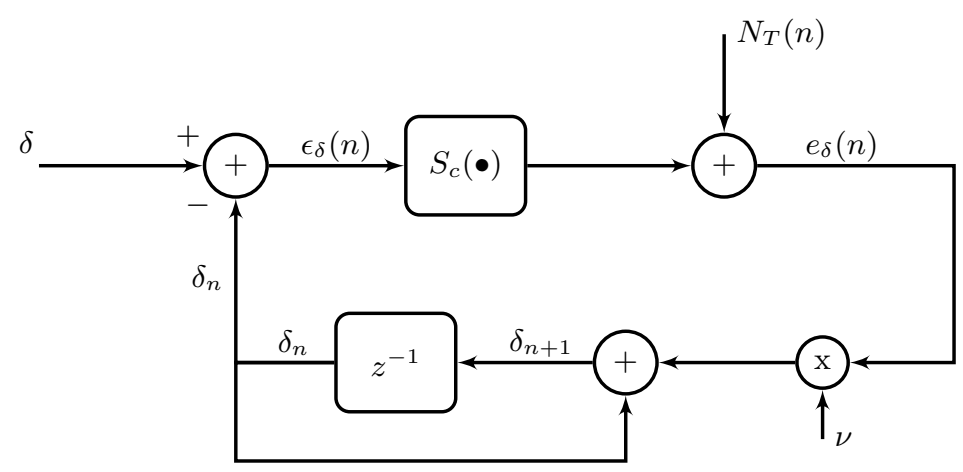

Fig. 3: Equivalent scheme of the FD estimation algorithm

The error signal $e_{\delta}(n)$ can be decomposed to the sum of the conditional expectation $E\left[e_{\delta}(n) \mid \delta(n)\right]$ and an additional random zero mean term. The first term is denoted S-curve and is function of the FD estimation error $\epsilon_{\delta}(n)=$ $\delta-\delta(n)$ and the second term is called loop noise and is denoted $N_{T}(n)$.

$$
\begin{aligned}
S_{c}\left(\epsilon_{\delta}(n)\right) & =E\left[e_{\delta}(n) \mid \delta(n)\right] \\
N_{T}(n) & =e_{\delta}(n)-S_{c}\left(\epsilon_{\delta}(n)\right)
\end{aligned}
$$

Based on the proposed FD algorithm (6)-(10), the S-curve can be expressed

$$
\begin{aligned}
S_{c}(\delta, \delta(n))= & P_{\beta_{\mathrm{T} \times \mathrm{L}}}[-\operatorname{sinc}(\delta)+\operatorname{sinc}(1-\delta) \\
& \left.+\operatorname{sinc}\left(\delta_{n}\right)-\operatorname{sinc}\left(1-\delta_{n}\right)\right] \sigma_{T x}^{4}
\end{aligned}
$$

with $\operatorname{sinc}(x)=\sin (\pi x) /(\pi x), P_{\beta_{\mathrm{T} \times \mathrm{L}}}=\left|\beta_{\mathrm{TxL}}\right|^{2}$ the power of the TxL channel, that is assumed to be known. In the context of small error of the FD estimation process, it is possible to linearize the S-curve around its stable equilibrium point [17]:

$$
S_{c}\left(\epsilon_{\delta}(n)\right) \approx \mathrm{D} \times \epsilon_{\delta}(n)
$$

where $D=P_{\beta_{T \times L}} \times \sigma_{T x}^{4}$ (with $\sigma_{T x}^{2}$ the variance of the transmitted samples) is the slope of the S-curve at the stable equilibrium point. (14) has been obtained using the approximation $\operatorname{sinc}(x) \approx 1-2 x$ for small $x$. As $D>0$, it shows that the FD estimator is stable, and converges to $\delta$ (i.e. the loop will lock within the range of FD). Besides, as $S_{c}(0)=0$, the FD estimator is unbiased. Assuming a white loop noise for small FD error, the variance of the FD estimation stage can be derived as [16]:

$$
\sigma_{\epsilon_{\delta}}^{2}=\frac{\nu}{\mathrm{D}(2-\nu \mathrm{D})} \Gamma_{N_{T}}[0]
$$

with $\Gamma_{N_{T}}[0]$ the loop noise auto-correlation at zero delay, that can be expressed as

$$
\Gamma_{N_{T}}[0]=\sigma_{\mathrm{Tx}}^{2} P_{\beta_{\mathrm{Tx}}}\left(\sigma_{x}^{2}+\sigma_{b}^{2}\right),
$$


From (15), (14) and (16) and under the aforementioned assumptions, the variance of the FD estimation process can finally be approximated as

$$
\sigma_{\epsilon_{\delta}}^{2} \approx \frac{\nu\left(\sigma_{x}^{2}+\sigma_{b}^{2}\right)}{4\left[1-\nu \sigma_{\mathrm{Tx}}^{4} P_{\beta_{\mathrm{TxL}}}\right]}
$$

Using the performance in terms of Signal to Interference Ratio (SIR) of the complex gain estimator (assuming a constant TxL channel) derived in presence of a non compensated FD are described in [7], we can now express the performance of the JE algorithm, using the variance of the FD estimator expressed in (17) :

$$
\begin{aligned}
\operatorname{SIR}_{\mathrm{comp}} \approx-10 \log _{10} & {\left[\frac{\mu \sigma_{u}^{2}}{2-\mu \sigma_{u}^{2}}++\left|\beta_{0_{\mathrm{Tx}} \mathrm{L}}\right|^{2} \frac{\sigma_{\epsilon_{\delta}}^{2}}{\sigma_{x_{b}}^{2}}\right.} \\
& \left.+\frac{\mu \sigma_{u}^{2}\left(\left|\beta_{0_{\mathrm{Tx \textrm {L }}}}\right|^{2}\right) \sigma_{\epsilon_{\delta}}^{2}}{\sigma_{x_{b}}^{2}\left(2-\mu \sigma_{u}^{2}\right)}\right]
\end{aligned}
$$

\section{Simulations}

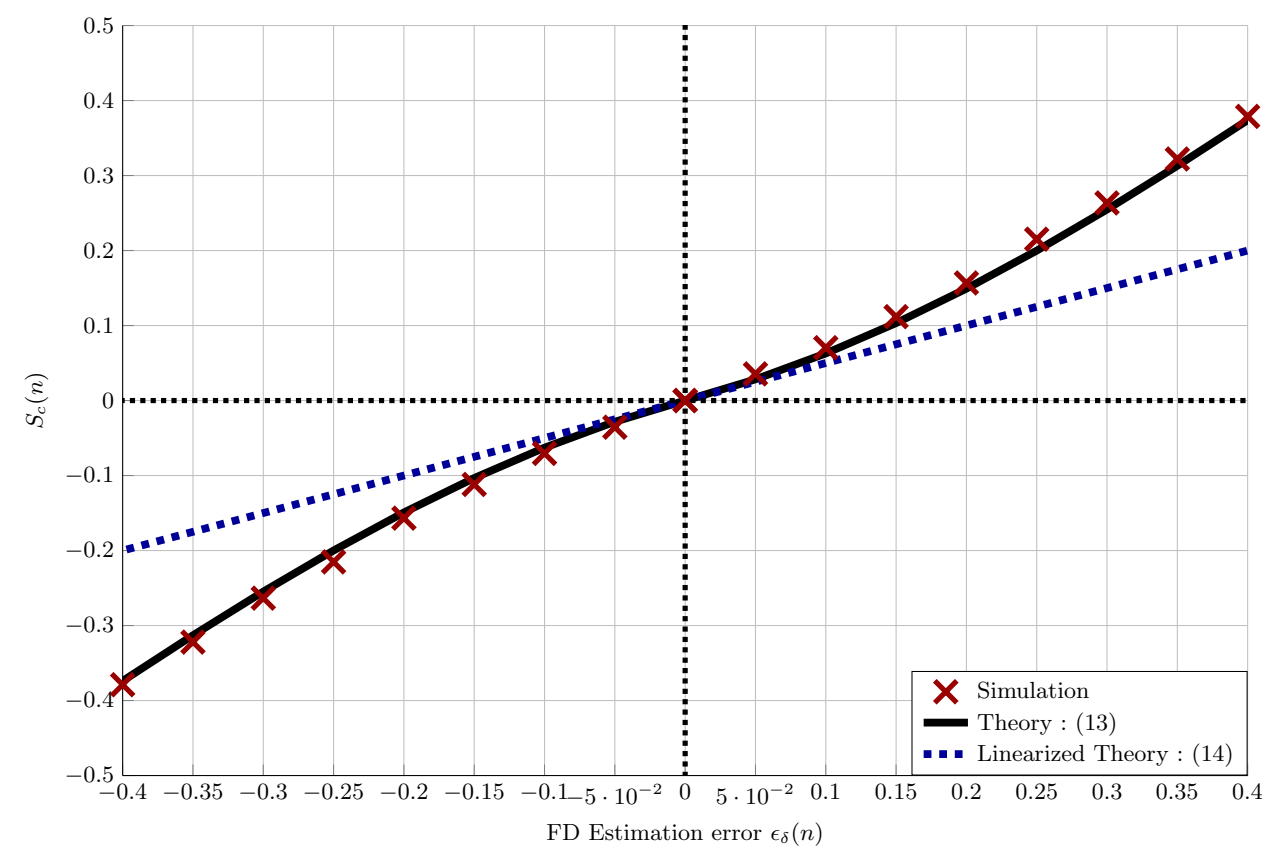

Fig. 4: Theoretical, simulated and linearized S-curve versus the FD estimation error

The performance of the proposed algorithm, and more precisely the performance of the FD algorithm are further analyzed by simulations. 


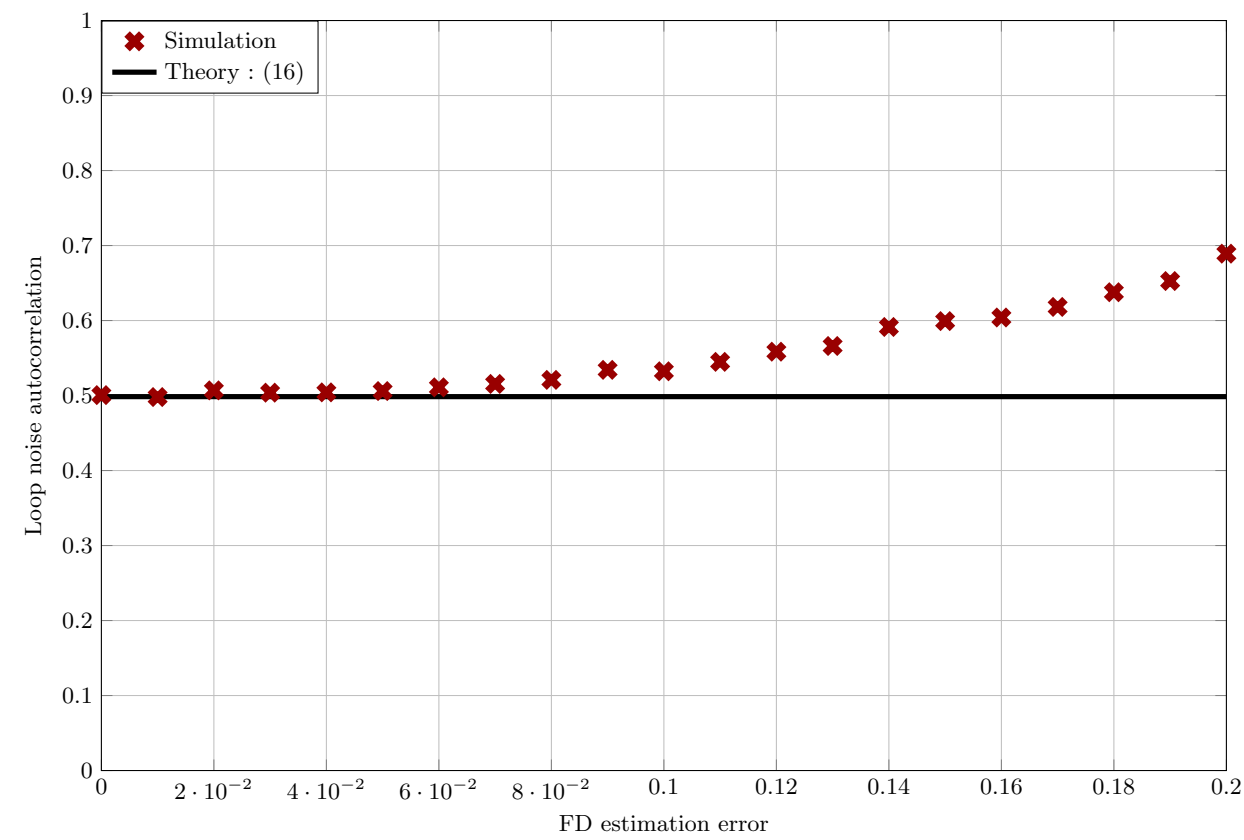

Fig. 5: Theoretical and simulated zero-delay autocorrelation of loop noise versus estimation error of the FD estimator

We first plot on the figure 4 the theoretical, the linearized theoretical and simulated S-curve versus the estimation error of the FD estimator in open loop. It is shown that the theory is corroborated and shows that the S-curve can be linearized when the estimation error is low (between -10 and 10 percent of the sampling time). It is also shown that the FD estimation loop will lock and is unbiased as $S_{c}(0)=0$.

On figure 5, we represent the theoretical and simulated loop noise zero-delay auto-correlation $\Gamma_{N_{T}}[0]$ versus the FD estimation error. It is shown that when the FD estimation error is low, the assumption of a white loop noise with variance expressed in (16) leads to accurate results.

We finally consider the performance of the JE algorithm on the figure 6 . We consider a white unitary variance desired noisy signal polluted by a TxL signal that follows (3). The interference level is set to $0 \mathrm{~dB}$ and the power of the desired noisy signal is set to $-80 \mathrm{~dB}$. The complex gain algorithm is piloted by a non interpolated reference $u(n)$ defined with (4) and the JE algorithm uses a Farrow structure to apply the estimated FD to $u(n)$. We also assume a constant TxL channel, and we represent the performance of the complex gain estimator and the JE algorithm for several values of FD. It is shown that the performance can be dramatically reduced if the FD algorithm is not enable, and secondly that the JE algorithm greatly improves the asymptotic performance. Is is also shown that the asymptotic performance of the structure can be approximated with (18) 


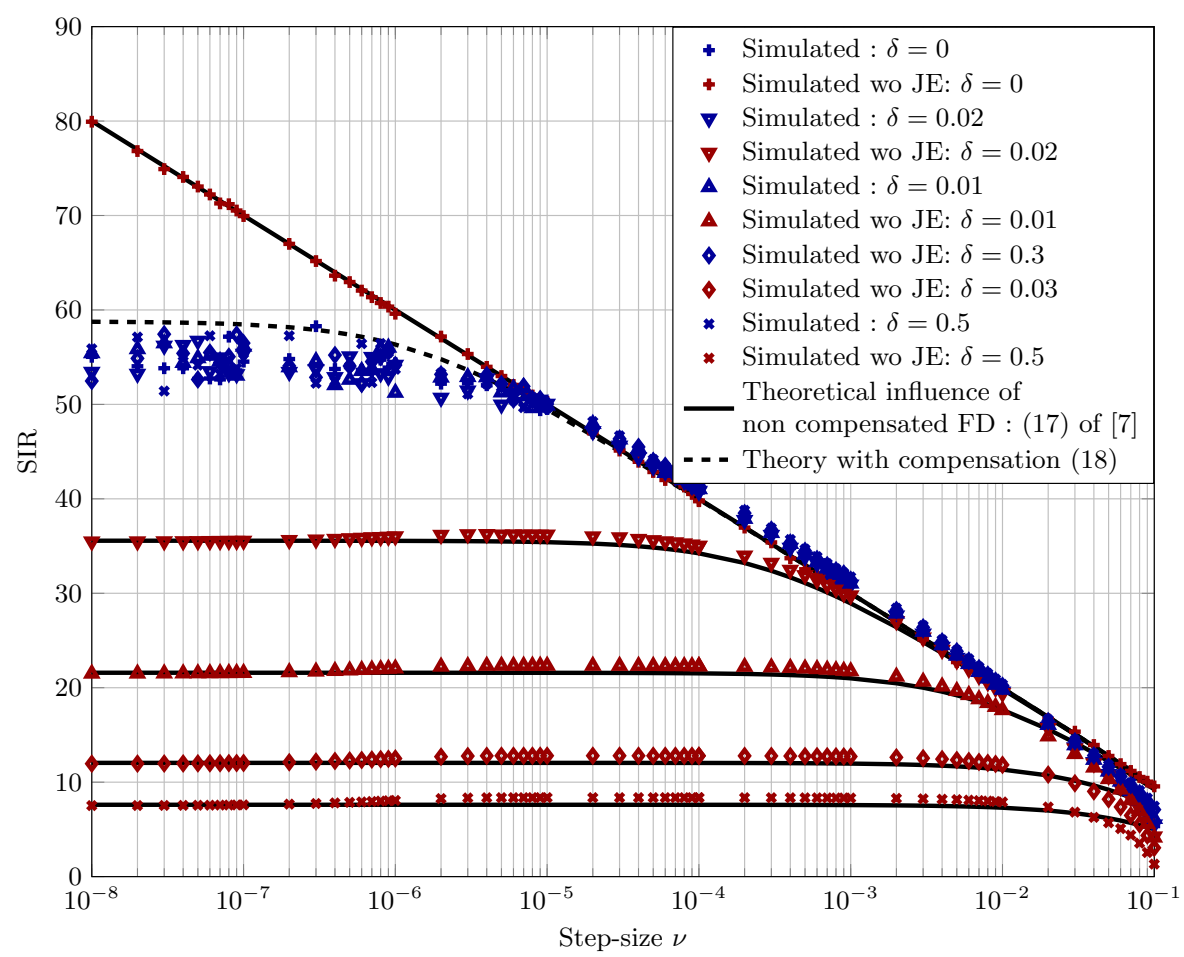

Fig. 6: Asymptotic performance of the complex gain estimator and the JE algorithm for several values of the step-size $\nu$ and for different values of fractional delays.

leading to a direct link between a desired asymptotic level and step-size value for the FD estimator $\nu$.

\section{Conclusion}

This paper deals with the performance of a joint estimation algorithm dedicated to the compensation of the digital Tx Leakage in RF transceivers. The strong constraints that apply in cognitive radios lead to a potential detrimental loss of performance due to hardware impairments and we focus here on a pollution that occurs in FDD transceivers, related to the pollution of an image of the transmitter stage on the receiver stage. Based on the initial algorithm proposed in [7], we focus in this paper on the fractional delay estimation algorithm that can be considered as a synchronisation algorithm piloted by an interpolated reference signal. We first have derived the analytical S-curve, defined as the conditional expectation of the error signal that controls the loop, and we have deduced an approximation of the asymptotic estimation error variance of the FD algorithm. This result can be used to properly tune the JE algorithm stepsize as the proposed approximated theoretical performance formula of the whole 
JE algorithm shows good accordance with simulations. As a perspective to this work, an implementation on a FDD device (or software defined radio) would be useful in order to validate the method and the theoretical results on experimental measurements.

\section{References}

1. D. Cabric and R. Brodersen. Physical layer design issues unique to cognitive radio systems. In Proc. IEEE 16th International Symposium on Personal, Indoor and Mobile Radio Communications (PIMRC), volume 2, pages 759-763 Vol. 2, Sept 2005.

2. K. Dufrene and R. Weigel. Highly linear IQ downconverter for reconfigurable wireless receivers. In The European Conference on Wireless Technology, pages 19-22, Oct 2005.

3. C. W. Farrow. A continuously variable digital delay element. In IEEE International Symposium on Circuits and Systems, pages 2641-2645 vol.3, 1988.

4. M. Faulkner. DC offset and IM2 removal in direct conversion receivers. IEE Proceedings on Communications, vol 149(3):pp 179-184, 2002.

5. G. Fettweis, M. Lohning, D. Petrovic, M. Windisch, P. Zillmann, and W. Rave. Dirty RF: a new paradigm. In Proc. IEEE 16th International Symposium on Personal, Indoor and Mobile Radio Communications (PIMRC), pages 2347-2355 Vol. 4, 2005.

6. A. Frotzscher and G. Fettweis. A stochastic gradient LMS algorithm for digital compensation of Tx leakage in Zero-IF-Receivers. In Proc. IEEE Vehicular Technology Conference (VTC), pages 1067-1071, 2008.

7. R. Gerzaguet, L. Ros, F. Belveze, and J.-M. Brossier. Joint estimation of complex gain and fractional delay for Tx leakage compensation in FDD transceivers. In Proc. 21st International Conference on Electronics Circuits and Systems (ICECS), 2014.

8. R. Gerzaguet, L. Ros, F. Belveze, and J.-M. Brossier. Performance of a digital transmitter leakage LMS-based cancellation algorithm for multi-standard radiofrequency transceivers. Digital Signal Processing, 51:35 - 46, 2016.

9. R. Gerzaguet, L. Ros, J.-M. Brossier, F. Belveze, and S. Ghandour-Haidar. Selfadaptive stochastic rayleigh flat fading channel estimation. In Proc. 18th International Conference on Digital Signal Processing (DSP 2013), pages 1-6, July 2013.

10. A. Kiayani, L. Anttila, and M. Valkama. Modeling and dynamic cancellation of Tx-Rx leakage in FDD transceivers. In Proc IEEE 56th International Midwest Symposium on Circuits and Systems (MWSCAS), pages 1089-1094, Aug 2013.

11. M. Knox. Single antenna full duplex communications using a common carrier. In Proc. IEEE 13th Annual Wireless and Microwave Technology Conference (WAMICON), pages 1-6, April 2012.

12. C. Lederer and M. Huemer. LMS based digital cancellation of second-order Tx intermodulation products in homodyne receivers. In Proc. IEEE Radio and Wireless Symposium (RWS), pages 207-210, 2011.

13. C. Lederer and M. Huemer. The influence of DC offsets on the digital cancellation of second-order Tx intermodulation distortions in homodyne receivers. In Proc. IEEE International Conference on Wireless Information Technology and Systems (ICWITS), pages 1-4, 2012. 
14. D. Manstretta, M. Brandolini, and F. Svelto. Second-order intermodulation mechanisms in CMOS downconverters. IEEE Journal of Solid-State Circuits, vol 38:pp 394-406, 2003.

15. H. Martikainen. Analysis of duplexing modes in the IEEE 802.16 wireless system. In Proc. European Wireless Conference (EW), pages 849-856, April 2010.

16. U. Mengali and A. D'Andrea. Synchronization Techniques for Digital Receivers. Applications of Communications Theory. Springer, 1997.

17. H. Meyr, M. Moeneclaey, and F. S.A. Digital Communication Receivers. Proakis, 1998.

18. B. Razavi. Design considerations for direct-conversion receivers. IEEE Transactions on Circuits and Systems (Analog and Digital Signal Processing), vol 44:pp 428-435, 1997.

19. L. Ros, H. Hijazi, and P. Simon, Eric. Complex Amplitudes Tracking Loop for multipath channel estimation in OFDM systems over slow to moderate fading. Signal Processing, 97(April):134-145, Apr. 2014.

20. E. Simon, K. Raoof, and L. Ros. Optimization of symbol timing recovery for multi-user DS-CDMA receivers. In Proc. IEEE of Acoustics, Speech, and Signal Processing (ICASSP), volume 4, pages IV-604-7 vol.4, April 2003.

21. H. Tang. Some physical layer issues of wide-band cognitive radio systems. In First IEEE International Symposium on New Frontiers in Dynamic Spectrum Access Networks (DySPAN), pages 151-159, Nov 2005. 